\title{
A Local Flow Phase Stretch Transform for Robust Retinal Vessel Detection
}

\author{
Mohsin Challoob and Yongsheng Gao \\ School of Engineering, Griffith University, Brisbane, Australia \\ mohsin. challoobdgriffithuni.edu.au, \\ yongsheng.gaodgriffith. edu. au
}

\begin{abstract}
This paper presents a new method for reliably detecting retinal vessel tree using a local flow phase stretch transform (LF-PST). A local flow evaluator is proposed to increase the local contrast and the coherence of the local orientation of vessel tree. This is achieved by incorporating information about the local structure and direction of vessels, which is estimated by introducing a second curvature moment evaluation matrix (SCMEM). The SCMEM evaluates vessel patterns as only features having linearly coherent curvature. We present an oriented phase stretch transform to capture retinal vessels running at various diameters and directions. The proposed method exploits the phase angle of the transform, which includes structural features of lines and curved patterns. The LFPST produces several phase maps, in which the vessel structure is characterized along various directions. To produce an orientation invariant response, all phases are linearly combined. The proposed method is tested on the publicly available DRIVE and IOSTAR databases with different imaging modalities and achieves encouraging segmentation results outperforming the state-of-the-art benchmark methods.
\end{abstract}

Keywords: Retinal vessel. Vessel detection. Local flow phase stretch transform

\section{Introduction}

The detection and examination of retinal vessel tree plays an essential role in clinical applications for the diagnosis of many eye diseases such as diabetic retinopathy $[1,2]$. The manual annotation of vessel network by graders is a laborious and time-consuming process due to the complexity of vessel structure. Automatic vessel segmentation can considerably decrease workload, enabling high throughput process in clinics. However, the automatic segmentation of vessel tree continues to be a challenging task because of many factors including low intensity contrast between vessel and background, variation of vessel width, uneven background illumination, and the presence of noise and pathological regions [3, 4]. Many segmentation algorithms have been introduced for the detection of retinal vessel tree [3-14]. Nevertheless, several challenges and limitations remain unsolved, as follows. First, the retinal vessel tree possesses a wide range of widths, where many vessels, particularly thin ones, are with a weak and unstable local intensity contrast and be overwhelmed in inhomogeneous background. If more 
attention is paid on the detection of the low contrast thin vessels, the wide vessels may be over-enhanced, and some false positive vessels may be generated. In contrast, when more emphasis is placed on wide vessels, poor contrast vessels are more likely to be lost. Secondly, besides vessel tree, a retinal image includes optic disc and may have pathological regions. Many of existing segmentation methods suffer from the falsepositive detections due to the presence of such regions. Further, a very limited number of methods has been developed for detecting vessel tree in scanning laser ophthalmoscopy (SLO) image. The majority of the existing segmentation algorithms only work for detecting retinal vessels in fundus image but fails to perform when applied on SLO images. Detecting retinal vessels in a SLO image encounters multiple difficulties such as strong light reflex, high curvature changes, more background artefacts, and many dark regions because of low light exposure during the image acquisition, making the segmentation process more challenging.

In this paper, a novel segmentation method for retinal vessel detection using a local flow phase stretch transform (LF-PST) is presented. A local flow evaluator is introduced to perform as an enhancing filter for increasing the local contrast and the coherence of the local orientation of retinal vessel tree. This is obtained by guiding the partial differential derivatives of gaussian smoothed vessel map with the orientation estimated by a second curvature moment evaluation matrix. The phase stretch transform (PST) is adapted to be an orientation-sensitive method and is employed for the detection of retinal vessels. We utilize the phase angle of the oriented PST for its functionality of being a detector for features like curved structures, and its brightness level equalization property. The LF-PST detects retinal vessels over multiple orientations to capture different vessel widths, producing several directional vessel maps. The final vessel tree is computed by fusing all directional phases of LF-PSTs to obtain a rotationally invariant response. The proposed method is assessed on different imaging modalities. Encouraging experimental results demonstrate the ability of the proposed method to work on different imaging modalities (fundus and SLO). This validates the performance stability of the proposed method against the state-of-the-art algorithms, which tend to be degraded when applied on SLO images.

The rest of the paper is organized as follows. The proposed method is introduced and explained in detail in Section 2. In Section 3, experimental results are conducted, and compared with other methods, while the discussion and conclusion are presented in Section 4.

\section{Proposed Method}

In this paper, a new method for reliably detecting retinal vessel tree is introduced using a local flow phase stretch transform (LF-PST). First, the green channel of a retinal image to be analyzed is used and converted to grey scale image because of its better vesselbackground intensity contrast. Then, the morphologically enhanced approach with disk-shaped structuring element [10] is applied to pre-process the grey scale image. The LF-PST is explained in detail as follows. 


\subsection{Local Flow Evaluator}

A local flow evaluator (LFE) is introduced to provide vessel tree with reliable information about local shape and direction of vessels, which improves the local contrast of vessel structure and the coherence of its local orientation. To achieve this, the partial differential derivatives (PDDs) of gaussian smoothed vessels is guided with the orientation estimated by a proposed second curvature moment evaluation matrix (SCMEM). The SCMEM utilizes the second moment matrix (SMM) used in studies $[15,16]$ as a local descriptor for flow-like structures, and the curvature evaluation introduced in [17] for measuring vessel-like structure by a gaussian profile whose curvature differs along the crest line. Fusing the curvature evaluation with the SMM can lead to stabilizing the directional behavior of the LFE by making the proposed SCMEM immune to noise while more sensitive to changes in orientation, producing robust information about vessel topology and its orientation. To implement our LFE, let $I(i, j)$ be an input image of finite size $M \times N$, where $0 \leq i \leq M-1$ and $0 \leq j \leq N-1$, the proposed SCMEM is calculated as follows:

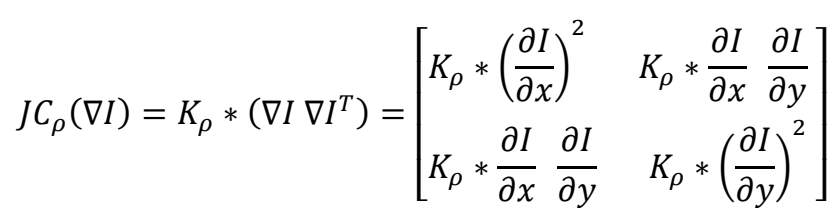

where $J C_{\rho}$ denotes the SCMEM and $K_{\rho}$ is the curvature evaluation represented by Laplacian of gaussian function with standard deviation $\rho=5$, where the sign of the curvature is approximated by the sign of the Laplacian. The $J C_{\rho}$ includes two orthonormal eigenvectors. The first vector $v_{1} \| \nabla I$ points to the direction of the gradient, whereas the second vector $v_{2} \| \nabla I^{T}$ refers to the direction of the level lines. In this paper, the second vector is normalized and only considered for estimating the local orientation of vessels. The second directional derivative $V_{w w}$, which is steered with orientation determined by $J C_{\rho}$, is calculated as:

$$
V_{w w}=c^{2} v_{x x}+c s v_{x y}+s^{2} v_{y y}
$$

where $c$ and $s$ are the first and second elements of the normalized eigenvector respectively, and $v_{x x}, v_{x y}$ and $v_{y y}$ are the second order derivatives of gaussian smoothed vessels with standard deviation $\sigma=2.5$. The LFE output $\left(E n h_{L F}\right)$ is defined as:

$$
\operatorname{Enh}_{L F}(i, j)=I(i, j)+H_{c}\left(V_{w w}(i, j)\right) * G(i, j)
$$

where $G=|\nabla I|$, and $H_{c}()$ is a continuous ramp function defined as $H_{c}\left(V_{w w}\right)=$ $-\left(1+\tanh \left(V_{w w}-\varepsilon\right)\right)$. The $\tanh ()$ is the hyperbolic tangent function and the parameter $\varepsilon$ controls the sensitivity of vessel detection. Larger values of $\varepsilon$ make the vessel detection less sensitive, while smaller values increase the detection sensitivity (in our experiments it is set to 0.5 ). 


\subsection{Local Flow Phase Stretch Transform}

Phase stretch transform (PST) is a physics-inspired approach emulating the propagation of light through a diffractive channel with warped dispersive characteristic, which was originally introduced as a photonic time stretch method in $[18,19]$. It has recently been used for the enhancement of impaired images in [20] with an orientation-insensitive kernel as shown in Figure 1. In this paper, the PST kernel is adapted to be an orientation-sensitive as illustrated in Figure 2. The LF-PST applies a 2-D nonlinear phase kernel to an input data in frequency domain. The amount of the phase assigned to the input relies on frequency, where higher amount of phase is applied to higher frequency features of the input image. According to [20], we exploit two unique properties from the PST for the vessel detection. The first is that the output of phase angle is corresponding to the even-order derivatives of input data with weighting factors. The key is the even order image derivatives encode shape information about local structures, detecting different image features. Also, the weighting factor is related to the assigned frequency, which can be selected to emphasize various structures of interest. The other property is that the output phase is inversely related to the brightness level of the input image. This crucial characteristic makes the transform more effective for the detection of structures in dark regions by equalizing input image brightness. The output of the LF-PST, represented by $\operatorname{LFPST}\left(i, j, \theta_{d}\right)$, is its phase in spatial domain and is defined as:

$$
\operatorname{LFPST}\left(i, j, \theta_{d}\right)=\Varangle\left\langle\operatorname{IFFT2}\left\{\operatorname{PK}\left(p, q, \theta_{d}\right) \cdot \operatorname{FFT} 2\left\{\operatorname{Enh}_{L F}(i, j)\right\}\right\}\right\rangle
$$

where $\measuredangle\langle$.$\rangle denotes the phase angle operator, FFT2 and IFFT2 are two-dimensional$ fast Fourier transform and its inverse, respectively, and $P K\left(p, q, \theta_{d}\right)$ is the phase kernel with 2-D frequency variables $(p$ and $q)$ and an orientation $\left(\theta_{d}\right)$. The $P K\left(p, q, \theta_{d}\right)$ is computed using a nonlinear frequency dependent phase $\varphi\left(p, q, \theta_{d}\right)$ as:

$$
\begin{gathered}
P K\left(p, q, \theta_{d}\right)=e^{j \varphi\left(p, q, \theta_{d}\right)} \\
\varphi\left(p, q, \theta_{d}\right)=\varphi_{\text {polar }}\left(r, \theta_{p}\right)=F(r) O\left(\theta_{p}\right) \\
F(r)=S_{t} \cdot \frac{W \cdot r \cdot \tan ^{-1}(W \cdot r)-(1 / 2) \cdot \ln \left(1+(W \cdot r)^{2}\right)}{W \cdot r_{\text {max }} \cdot \tan ^{-1}\left(W \cdot r_{\max }\right)-(1 / 2) \cdot \ln \left(1+\left(W \cdot r_{\max }\right)^{2}\right)} \\
O\left(\theta_{p}\right)=\cos ^{2}\left(\theta_{T}\right) \cdot H_{s}\left(\frac{\theta_{T}}{\pi}+\frac{1}{2}\right) \cdot H_{s}\left(\frac{1}{2}-\frac{\theta_{T}}{\pi}\right) \\
\theta_{T}=\left|\tan ^{-1}\left(\frac{\sin \left(\tan ^{-1}\left(\frac{q}{p}\right)\right) \cdot \cos \left(\theta_{d}\right)-\cos \left(\tan ^{-1}\left(\frac{q}{p}\right)\right) \cdot \sin \left(\theta_{d}\right)}{\cos \left(\tan ^{-1}\left(\frac{q}{p}\right)\right) \cdot \cos \left(\theta_{d}\right)+\sin \left(\tan ^{-1}\left(\frac{q}{p}\right)\right) \cdot \sin \left(\theta_{d}\right)}\right)\right|
\end{gathered}
$$

where $F(r)$ and $O\left(\theta_{p}\right)$ are frequency and angular components of the phase kernel, respectively, $r=\sqrt{p^{2}+q^{2}}$, and $r_{\max }$ is the maximum frequency. The $\tan ^{-1}()$, $\sin ()$, and $\cos ()$ are the inverse tangent, sine and cosine functions, respectively, 
whereas $\ln ()$ and $H_{s}$ are the natural logarithm and heaviside step functions, respectively, and the parameters $S_{t}$ and $W$ are the strength and wrap of the phase profile. These parameters $\left(S_{t}\right.$ and $\left.W\right)$ control the amount of the phase applied to each frequency at the input image, and they are set to be unity, which have no impact on obtained results, making the amount of phase applied the main parameter in this paper. This in turn reduces the complexity in determining the LF-PST parameters. The angular component $O\left(\theta_{p}\right)$ is preforming as a filter to determine the orientation for where the kernel of the LF-PST is applied, and this filter is designed by the rotation matrix and cosine function, which is with a fixed bandwidth of $\pi / 2$ radius.
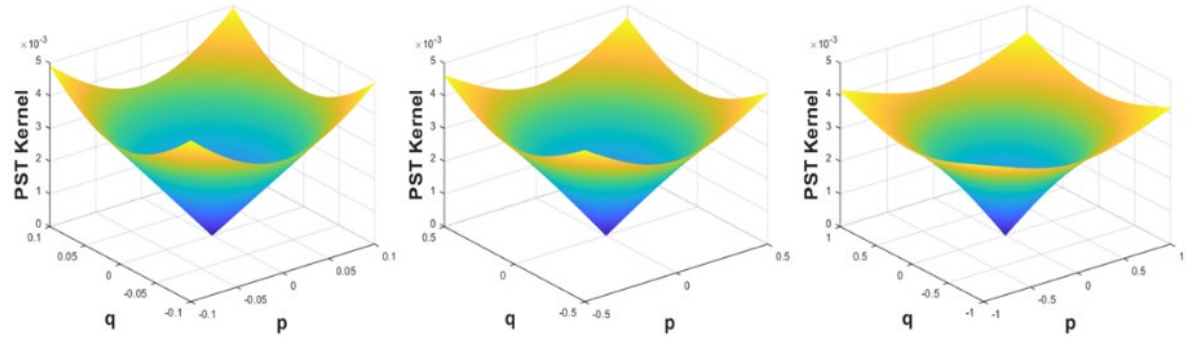

Fig. 1. The gradient of orientation-insensitive PST kernels with various frequencies $(0.1,0.5,1$, respectively).
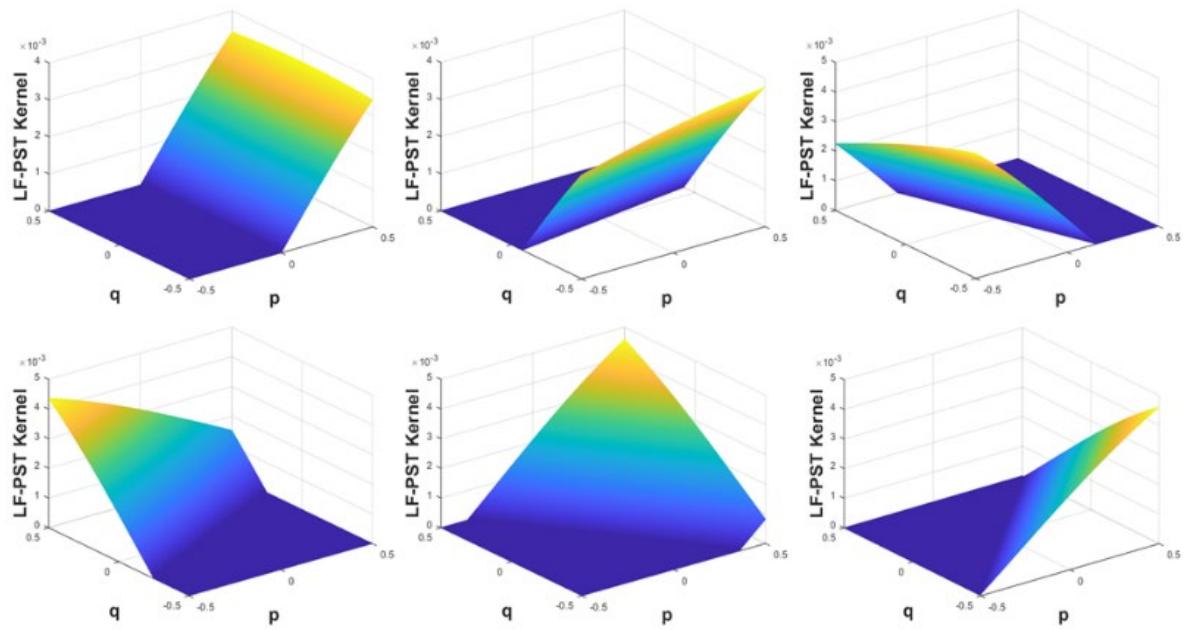

Fig. 2. The gradient of orientation-sensitive LF-PST kernels with various angles: first row $(\pi, \pi / 6,2 \pi / 6)$ and second row $(3 \pi / 6,4 \pi / 6,5 \pi / 6)$, respectively. 


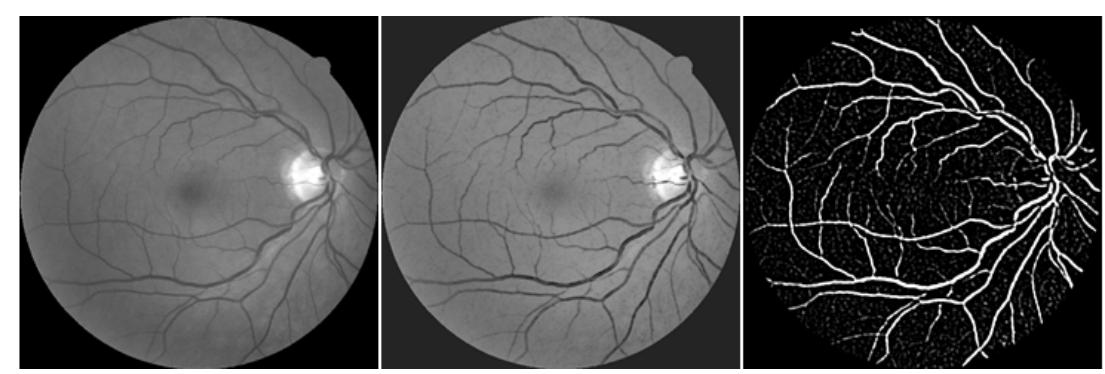

Fig. 3. Original image, LFE response, and final response of LF-PST, respectively.

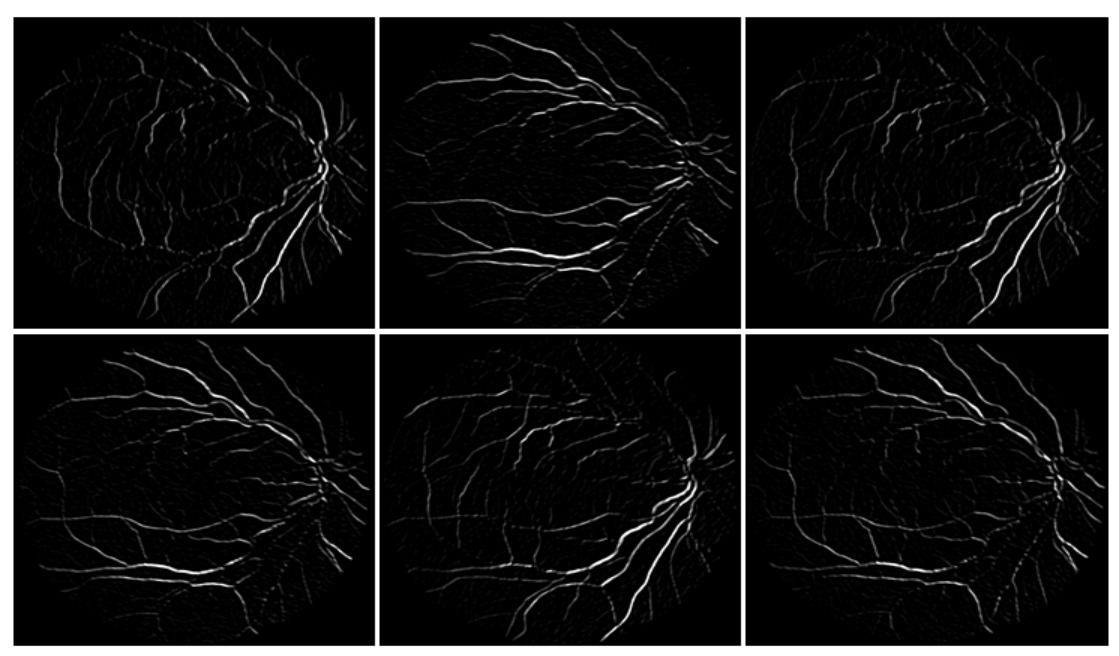

Fig. 4. Output phases of LF-PSTs with various orientations : first row with angles $(\pi, \pi / 6$, $2 \pi / 6)$ and second row with angles $(3 \pi / 6,4 \pi / 6,5 \pi / 6)$, respectively.

The final vessel tree $\left(F_{\text {vess }}\right)$, which is illustrated in Figure 3, is produced by combining all phases of the LF-PSTs linearly as:

$$
F_{\text {vess }}(i, j)=\sum_{\theta_{d} \in \Theta} \operatorname{LFPST}\left(i, j, \theta_{d}\right)
$$

where $\Theta$ denotes a set of angles with consideration: $\Theta=\{\pi, \pi / 6,2 \pi / 6,3 \pi / 6,4 \pi / 6$, $5 \pi / 6\}$. Figure 4 demonstrates a set of directional vessel tree generated with $\Theta$. As a postprocessing step, the local entropy thresholding approach is employed to threshold the vessel tree response $\left(F_{\text {vess }}\right)$, where the co-occurrence matrix is used to compute the local entropy for obtaining an optimal threshold value. 


\section{Results}

The performance of proposed method is evaluated on two publicly available DRIVE and IOSTAR databases. The DRIVE contains fundus images, and its test set (20 images) with ground truth A (1st_manual) is used for our experiments. The IOSTAR database consists of 30 images acquired with a scanning laser ophthalmoscopy (SLO) technology. The proposed method is quantitively measured using three commonly used metrics: accuracy (ACC), specificity (SP) and sensitivity (SE). The proposed method is performed on a PC with Intel Core i5, 3.40GHz, 8.00GB RAM using MATLAB R2018b, consuming in average 3.9 seconds on a DRIVE image and 9.3 seconds on an IOSTAR image. The amount of the applied phase is experimentally examined as shown in Figure 5 by using four frequency values $(0.1,0.5,1,1.5)$ on both databases. When a small frequency is used, more vessel pixels are detected, but detection results are more sensitive to noise and non-vessel interfaces. Also, the wide vessels, especially the ones with central light reflex, are falsely detected as two small vessels. In contrast, when a large frequency is assigned, more vessels are left undetected, close vessels are merged together, and wide vessels become dilated . Based on these experiments, the amount of phase applied to the DRIVE images is set to 0.5, while for the SLO images it is set to 1. Figure 6 illustrates the segmentation results obtained by the proposed algorithm.

The performance of the proposed method is compared with eleven state-of-the-art algorithms using the average values of the used metrics (see Table 1), including supervised methods [6,7] and unsupervised ones $[3,4,5,8,9,11,12,13,14]$. To have fair comparison, the proposed method is assessed against the methods that used the same ground truth as our method, so the results of paper [3] on the DRIVE are excluded from Table 1 due to the use of the second observer results as ground truth. The results of the methods [11-14] on the IOSTAR database are taken from the published results in [3] and the results of methods [6] and [11] on the DRIVE are from [9] and [21], respectively. The rest are obtained from the published results in their original papers. As illustrated in Table 1, the proposed method achieves encouraging results. For the IOSTAR database, the proposed method obtains the highest average accuracy and specificity against other methods. Also, it produces higher sensitivity than the methods in $[11,13]$. For the DRIVE database, the proposed method produces higher accuracy than other algorithms except $[5,14]$, higher sensitivity than the methods of $[5,6,7,11,13]$, and higher specificity than the methods of $[4,12,13]$. The proposed method is also qualitatively evaluated with two benchmark methods $[17,22]$ on an example image from the IOSTAR database (see Figure 7). The benchmark methods enhance background region, making identifying vessel tree difficult, showing their weaknesses in the detection of retinal vessel tree in the presence of high curvature changes, more background artefacts, and large dark regions.

\section{Discussion and Conclusion}

In general, it is challenging to design a vessel detection method to be feasible across different imaging modalities. Also, a very few algorithms have been introduced in 
literature to extract retinal vessel tree from SLO images in comparison with those ones presented for fundus images, where the SLO image is generally associated with problems of strong light reflex, high curvature changes, and many dark regions. According to the achieved results, the proposed method demonstrates its reliability to detect different vessel width classes (wide, median and fine) and its stability to work on two dissimilar imaging modalities (fundus and SLO).

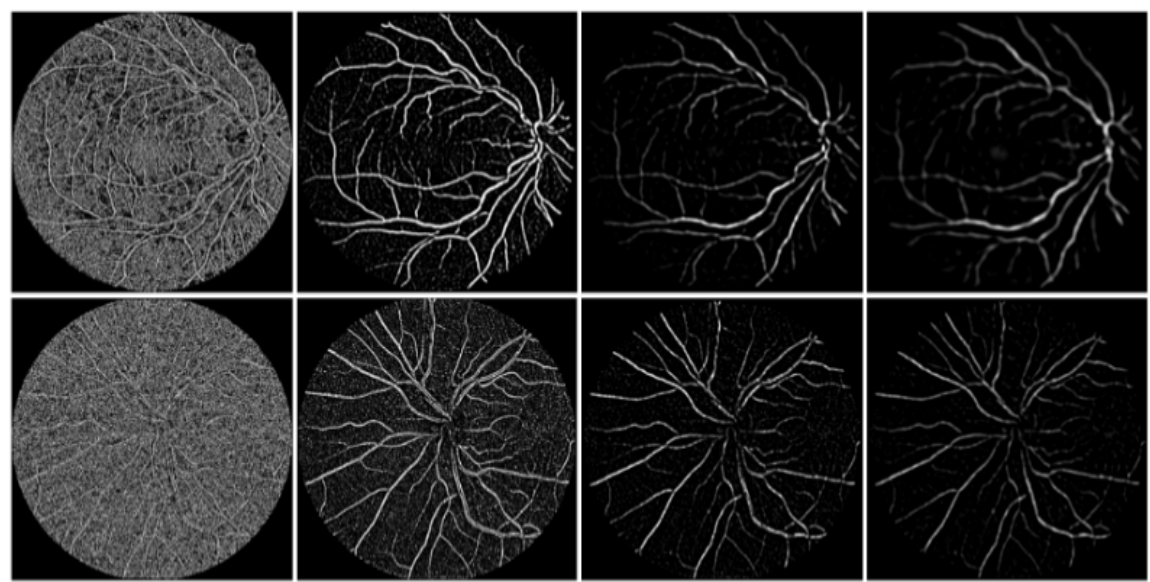

Fig. 5. The LF-PST responses. First row: four frequencies $(0.1,0.5,1,1.5)$ applied on a DRIVE image, respectively. Second row: the same frequencies applied on a SLO image, respectively.
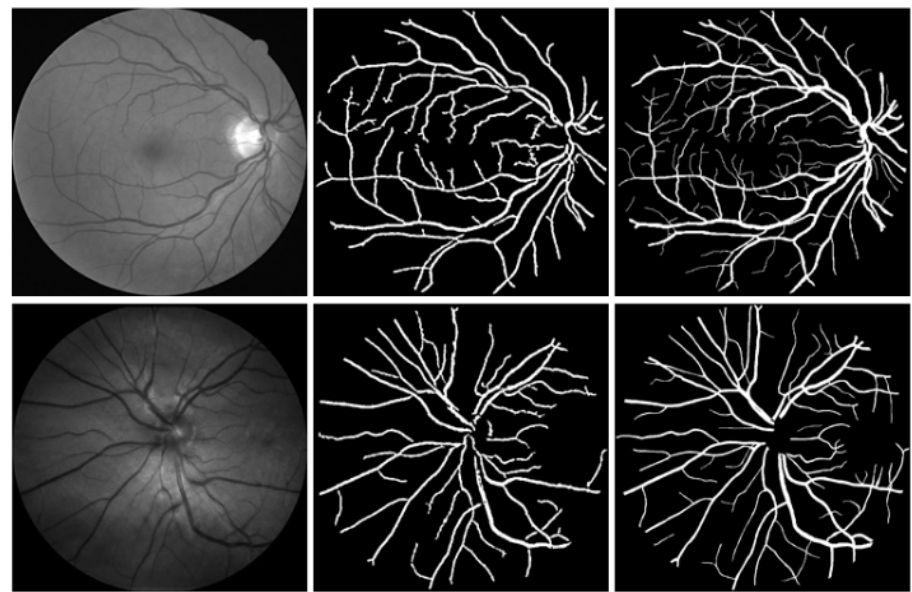

Fig. 6. First column: original images from DRIVE and IOSTAR datasets, respectively. Second column: segmentation results of the proposed method. Third column: ground truth 
Table 1. Performance comparison of the proposed method against benchmark methods.

\begin{tabular}{|l|l|l|l|l|l|l|}
\hline Database & \multicolumn{3}{|c|}{ IOSTAR } & \multicolumn{3}{|c|}{ DRIVE } \\
\hline Method & ACC & SP & SE & ACC & SP & SE \\
\hline Zhao [3] & 0.948 & 0.967 & 0.772 & - & - & - \\
\hline Zhang [4] & 0.951 & 0.974 & 0.754 & 0.947 & 0.972 & 0.774 \\
\hline Sazak [5] & - & - & - & 0.959 & 0.981 & 0.718 \\
\hline Soares [6] & - & - & - & 0.944 & 0.976 & 0.723 \\
\hline Marín [7] & - & - & - & 0.945 & 0.980 & 0.706 \\
\hline Lam [8] & - & - & - & 0.947 & - & - \\
\hline Zhao [9] & - & - & - & 0.947 & 0.978 & 0.735 \\
\hline Frangi [11] & 0.920 & 0.931 & 0.704 & 0.933 & 0.975 & 0.646 \\
\hline Azzopardi [12] & 0.941 & 0.967 & 0.761 & 0.944 & 0.970 & 0.765 \\
\hline Bankhead [13] & 0.911 & 0.921 & 0.726 & 0.937 & 0.971 & 0.702 \\
\hline Zhao [14] & 0.928 & 0.935 & 0.757 & 0.953 & 0.978 & 0.744 \\
\hline Proposed Method & $\mathbf{0 . 9 5 7}$ & $\mathbf{0 . 9 7 5}$ & $\mathbf{0 . 7 5 0}$ & $\mathbf{0 . 9 5 2}$ & $\mathbf{0 . 9 7 4}$ & $\mathbf{0 . 7 3 2}$ \\
\hline
\end{tabular}

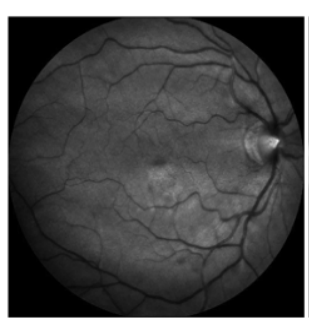

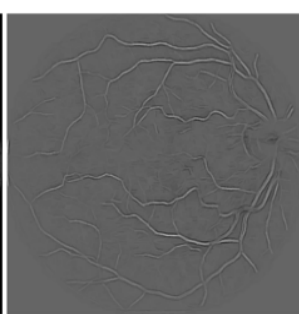

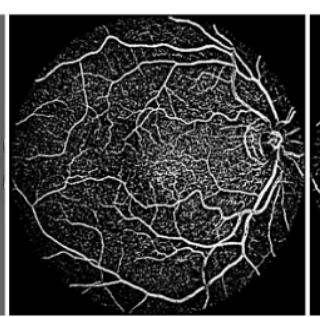

d

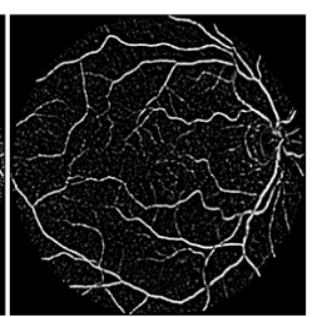

Fig. 7. Comparison of the proposed method against benchmark methods: (a) a randomly chosen IOSTAR image, (b) Zana [17], (c) Nguyen [22], (d) our method.

Also, the proposed method shows an effective performance over previously reported methods for segmenting SLO images by producing the highest accuracy and specificity. The segmentation results of many of previous reported methods are degraded when applied to the SLO image, as demonstrated in Table 1 and Figure 7. The integration of the curvature evaluation with the SMM results in stabilizing the directional behavior of the SCMEM, making the LFE immune to noise while more sensitive to changes in orientation particularly in the regions with high curvature changes. This is achieved by providing reliable information about local shape and direction of vessels, which increases the local contrast of vessel structure and the coherence of its local orientation. This in turn leads to preserving more vessels especially the ones with low intensity contrast while reducing the detection of false positives. Also, since retinal vessels can present in any orientation and be with various widths and lengths, the use of a set of the directional LF-PSTs whose outputs can be fusing linearly to cover the entire range of possible angles is necessary. This contributes to the detection of vessels with low contrast at different widths. The crucial properties of the PST of being a local shape 
detector and brightness level equalization makes the proposed method robust to detect vessels in the presence of high curvature changes, dim areas and inhomogeneous intensity. The LF-PST kernel includes a built-in logarithmic function, which equalizes the brightness in the input image by giving a higher gain for lower brightness and vice versa. The use of this function in the LF-PST improves the vessel detection results particularly in the SLO image, which has many dark regions. Also, the proposed method offers an advantage of the controllability over detection results via the amount of the applied phase in the LF-PST kernel with an angle, providing the proposed method with more flexibility to reduce false detection while retaining vessels, and to deal with the variability of vessel width. While we have shown the effectiveness of the proposed method on the detection of retinal vessels in two different imaging modalities, the LFPST is also feasible for the detection of crack tree in pavement images, see Figure 8, where encouraging results are achieved on pavement images suffering from the problems of noise, shadow, low contrast and occlusion.

In conclusion, this paper presents a new unsupervised method using the proposed local flow phase stretch transform (LF-PST) for the reliable detection of retinal vessel tree. The properties of the LF-PST of being an orientation-sensitive and a detector for line-like structures with its brightness equalization make its performance robust for the detection of retinal vessel tree in different imaging modalities, producing successful segmentation results, which outperforms the state-of-the-art benchmark algorithms. However, the main drawback of the proposed method is that it still produces the false detection around optic disc area. The directions for future work will include: 1) the development of 3D- LF-PST as there is an increase in the use of 3D vessel detection in medical clinics; 2) the extension of the proposed method to detect other curvilinear structures such as vein patterns in leaf images, and roads in aerial images.
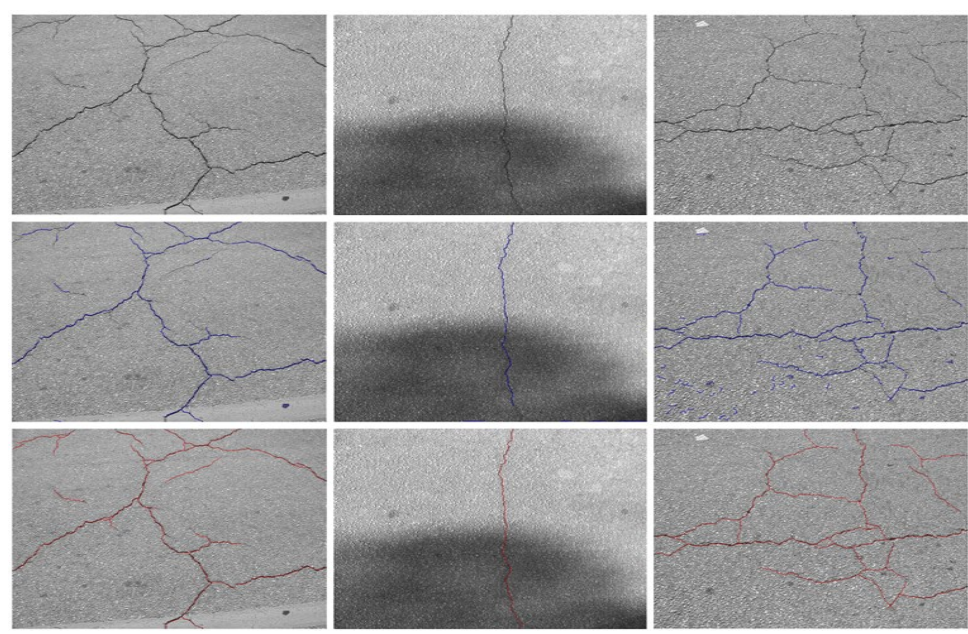

Fig. 8. First row: original pavement images taken from database in [23]. Second row: detection results of our method overlaid on original images with blue color. Third row: ground truths overlaid with red color. 


\section{References}

1. Bibiloni, P., González-Hidalgo, M., Massanet, S.: A survey on curvilinear object segmentation in multiple applications. Pattern Recognition. 60, 949-970 (2016). doi: 10.1016/j.patcog.2016.07.023

2. Annunziata, R., Garzelli, A., Ballerini, L., Mecocci, A., Trucco, E.: Leveraging Multiscale Hessian-Based Enhancement With a Novel Exudate Inpainting Technique for Retinal Vessel Segmentation. IEEE Journal of Biomedical and Health Informatics. 20, 1129-1138 (2016). doi: 10.1109/JBHI.2015.2440091

3. Zhao, Y., Zheng, Y., Liu, Y., Zhao, Y., Luo, L., Yang, S., Na, T., Wang, Y., Liu, J.: Automatic 2-D/3-D Vessel Enhancement in Multiple Modality Images Using a Weighted Symmetry Filter. IEEE Transactions on Medical Imaging. 37, 438-450 (2018). doi:10.1109/TMI.2017.2756073

4. Zhang, J., Dashtbozorg, B., Bekkers, E., Pluim, J., Duits, R., ter Haar Romeny, B.: Robust Retinal Vessel Segmentation via Locally Adaptive Derivative Frames in Orientation Scores. IEEE Transactions on Medical Imaging. 35, 2631-2644 (2016). doi: 10.1109/TMI.2016.2587062

5. Sazak, Ç., Nelson, C., Obara, B.: The multiscale bowler-hat transform for blood vessel enhancement in retinal images. Pattern Recognition. 88, 739-750 (2019). doi: 10.1016/j.patcog.2018.10.011

6. Soares, J., Leandro, J., Cesar, R., Jelinek, H., Cree, M.: Retinal vessel segmentation using the 2-D Gabor wavelet and supervised classification. IEEE Transactions on Medical Imaging 25, 1214- 1222 (2006). doi:10.1109/tmi.2006.879967

7. Marín, D., Aquino, A., Gegundez-Arias, M., Bravo, J.: A New Supervised Method for Blood Vessel Segmentation in Retinal Images by Using Gray-Level and Moment Invariants-Based Features. IEEE Transactions on Medical Imaging. 30, 146-158 (2011). doi: 10.1109/tmi.2010.2064333

8. Lam, B., Gao, Y., Liew, A.: General Retinal Vessel Segmentation Using RegularizationBased Multiconcavity Modeling. IEEE Transactions on Medical Imaging. 29, 1369-1381 (2010). doi: 10.1109/tmi.2010.2043259

9. Zhao, Y., Wang, X., Wang, X., Shih, F.: Retinal vessels segmentation based on level set and region growing. Pattern Recognition. 47, 2437-2446 (2014). doi: 10.1016/j.patcog.2014.01.006

10. Challoob, M., Gao, Y.: Retinal Vessel Segmentation Using Matched Filter with Joint Relative Entropy. International Conference on Computer Analysis of Images and Patterns. pp. 228-239. Springer (2017). doi: 10.1007/978-3-319-64689-3_19

11. Frangi, A., Niessen, W., Vincken, K., Viergever, M.: Multiscale Vessel Enhancement Filtering. International Conference on Medical Image Computing and Computer-Assisted Intervention. pp. 130-137. Springer (1998). doi: 10.1007/BFb0056195

12. Azzopardi, G., Strisciuglio, N., Vento, M., Petkov, N.: Trainable COSFIRE filters for vessel delineation with application to retinal images. Medical Image Analysis. 19, 46-57 (2015). doi: 10.1016/j.media.2014.08.002

13. Bankhead, P., Scholfield, C., McGeown, J., Curtis, T.: Fast Retinal Vessel Detection and Measurement Using Wavelets and Edge Location Refinement. PLoS ONE. 7, (2012). doi: 10.1371/journal.pone.0032435

14. Zhao, Y., Liu, Y., Wu, X., Harding, S., Zheng, Y.: Retinal Vessel Segmentation: An Efficient Graph Cut Approach with Retinex and Local Phase. PLoS ONE. 10, (2015). doi: 10.1371/journal.pone.0122332 
15. Vicas, C., Nedevschi, S.: Detecting Curvilinear Features Using Structure Tensors. IEEE Transactions on Image Processing. 24, 3874-3887 (2015). doi: 10.1109/tip.2015.2447451

16. Weickert, J.: Coherence-Enhancing Shock Filters. Joint Pattern Recognition Symposium. pp. 1-8. Springer (2003). doi: 10.1007/978-3-540-45243-0_1

17. Zana, F., Klein, J.: Segmentation of vessel-like patterns using mathematical morphology and curvature evaluation. IEEE Transactions on Image Processing. 10, 1010-1019 (2001). doi: $10.1109 / 83.931095$

18. Bhushan, A., Coppinger, F., Jalali, B.: Time-stretched analogue-to-digital conversion. Electronics Letters. 34, 839 - 841 (1998). doi: 10.1049/el:19980629

19. Han, Y., Jalali, B.: Photonic time-stretched analog-to-digital converter: fundamental concepts and practical considerations. Journal of Lightwave Technology. 21, 3085-3103 (2003). doi: $10.1109 /$ jlt.2003.821731

20. Suthar, M., Asghari, H., Jalali, B.: Feature Enhancement in Visually Impaired Images. IEEE Access. 6, 1407-1415 (2018). doi: 10.1109/access.2017.2779107

21. Cheng, E., Du, L., Wu, Y., Zhu, Y., Megalooikonomou, V., Ling, H.: Discriminative vessel segmentation in retinal images by fusing context-aware hybrid features. Machine Vision and Applications. 25, 1779-1792 (2014). doi: 10.1007/s00138-014-0638-x

22. Nguyen, U., Bhuiyan, A., Park, L., Ramamohanarao, K.: An effective retinal blood vessel segmentation method using multi-scale line detection. Pattern Recognition. 46, 703-715 (2013). doi:10.1016/j.patcog.2012.08.009

23. Zou, Q., Cao, Y., Li, Q., Mao, Q., Wang, S.: CrackTree: Automatic crack detection from pavement images. Pattern Recognition Letters. 33, 227-238 (2012). doi: 10.1016/j.patrec.2011.11.004 